\title{
Dark Solitons in High Velocity Waveguide Polariton Fluids
}

\author{
P. M. Walker, ${ }^{1, *}$ L. Tinkler, ${ }^{1}$ B. Royall, ${ }^{1}$ D. V. Skryabin, ${ }^{2,3}$ I. Farrer, ${ }^{4}$ \\ D. A. Ritchie, ${ }^{5}$ M. S. Skolnick, ${ }^{1}$ and D. N. Krizhanovskii ${ }^{1}$ \\ ${ }^{1}$ Department of Physics and Astronomy, University of Sheffield, S3 7RH Sheffield, United Kingdom \\ ${ }^{2}$ Department of Physics, University of Bath, BA2 7AY Bath, United Kingdom \\ ${ }^{3}$ Department of Nanophotonics and Metamaterials, ITMO University, Saint Petersburg 197101, Russia \\ ${ }^{4}$ Department of Electronic and Electrical Engineering, University of Sheffield, S3 $7 H Q$ Sheffield, United Kingdom \\ ${ }^{5}$ Cavendish Laboratory, University of Cambridge, CB3 OHE Cambridge, United Kingdom
}

(Received 21 March 2017; published 31 August 2017)

\begin{abstract}
We study exciton-polariton nonlinear optical fluids in the high momentum waveguide regime for the first time. We demonstrate the formation of dark solitons with the expected dependence of width on fluid density for both main classes of soliton-forming fluid defects. The results are well described by numerical modeling of the fluid propagation. We deduce a continuous wave nonlinearity more than ten times that on picosecond time scales, arising due to interaction with the exciton reservoir.
\end{abstract}

DOI: 10.1103/PhysRevLett.119.097403

Exciton polaritons are half-light half-matter quasiparticles resulting from strong coupling between photons and quantum-well (QW) excitons [1]. They behave like photons but experience nonlinearity at least 1000 times larger than in weakly coupled semiconductors due to exciton-exciton scattering [2]. In a waveguide geometry [3] the propagation of light is dominated by the high momentum $\beta$ and velocity in the propagation direction $z$. The envelope of the optical field evolves slowly compared to the wavelength $2 \pi / \beta$, which leads to its evolution equation becoming formally analagous to the nonlinear Schrodinger (NSE) or GrossPitaevskii (GPE) equations, but with $z$ playing the role of time [4]. This high-momentum paraxial regime has been exploited for photonic simulation of complex Hamiltonians [5-9]. The high velocity and ease of growth and fabrication also make waveguides promising for nonlinear polaritonic circuits, simulators, and studies of topological phases [10]. Among the most fundamental solutions of the GPE are dark solitons [11-21]. These are self-localized dark notches on an infinitely extended bright background accompanied by a phase jump at the center. In the field of nonlinear optics they offer potential applications in all-optical signal processing [22]. The giant polariton nonlinearity allows dark soliton formation at the submillimeter length scales needed for on-chip integration [19], a regime previously inaccessible due to weak photon-only nonlinearities.

In this work we experimentally study spatial dark polariton soliton formation in the high-momentum regime for the first time. We resonantly inject both main classes of

Published by the American Physical Society under the terms of the Creative Commons Attribution 4.0 International license. Further distribution of this work must maintain attribution to the author(s) and the published article's title, journal citation, and DOI. initial condition from which dark solitons form. A phase jump in the input field leads to the formation of a single soliton while an amplitude defect gives a pair of solitons with opposite phase jumps across their cores. Solitons are formed within $600 \mu \mathrm{m}$ at $\mathrm{cw}$ powers less than $30 \mathrm{~mW}$. In Bragg microcavities dark-solitonlike features were previously reported [19-21]. As discussed in Ref. [23] some of the signatures used to show polariton dark soliton formation may also occur in the linear regime for particular input conditions so that previous observations of polariton dark solitons have been the cause of controversy [23-25]. The self-localized nature of dark solitons arises from the balance of the kinetic energy associated with the localized defect, determined by its width, and the nonlinear potential energy, proportional to the density $n$ of the background (analogous to the case of quantized vortices in a polariton condensate [26]). Thus the unambiguous signature of dark solitons, which we demonstrate in this paper, is that the width should be determined by the density [23]. Quantitatively, the width decreases with increasing density according to Eq. (1), which follows from the analytical dark-soliton solution of the GPE [11,12,27],

$$
\left(X_{0} / 1.7627\right)^{2}=v_{\mathrm{g}, \mathrm{LP}} /(\beta g n)
$$

Here $X_{0}$ is the notch full width halfway between minimum and maxima, $v_{\mathrm{g}, \mathrm{LP}}=24 \mu \mathrm{mps}^{-1}$ and $\beta=23.7 \mu \mathrm{m}^{-1}$ are the polariton group velocity and wave number, and $\hbar g$ is the polariton-polariton interaction energy per unit polariton density. To establish soliton behavior, this width-density criterion should be evaluated over a range of densities and for multiple defect types to exclude coincidental similarity to soliton profiles in particular cases [25].

Compared to microcavities our waveguide is effectively one dimensional in the coordinate, $x$, transverse to the fluid 
flow, with $z$ being the evolution coordinate. As discussed above it operates in the paraxial regime and is described by qualitatively different physics to microcavities. Experimentally, the range of densities over which solitons can be studied in microcavities is limited because the fluid speed of sound, which increases with density, exceeds the flow velocity and prevents the nucleation of solitons [19] before the soliton width strongly deviates from its low density value [24]. The waveguide geometry allows injection of arbitrary initial density and field profiles, which then evolve into solutions of the GPE. The fluid velocity in waveguides (24 $\mu \mathrm{m} \mathrm{ps}^{-1}$ at this detuning) is at least ten times larger than in microcavities and so is always well above the sound velocity (up to $4.8 \mu \mathrm{m} \mathrm{ps}^{-1}$ at our highest densities [28]). We are therefore able to study the soliton core size varying over a wide range of background densities and verify the width-density criterion. The initial fields and hence dynamics in the linear regime are fully characterized so that the deviations caused by the nonlinearity are clear, providing conclusive proof for the generation of polariton dark solitons.

There is also still a great deal of experimental uncertainty over the precise nature and strength of the polariton nonlinearity that underlies polariton soliton formation, with estimates of the interaction strength varying over 3 orders of magnitude [29-35]. A quantitative understanding of these interactions is important as they underpin efforts towards polariton fermionization [36-38] and strongly quantumcorrelated states in polaritonic lattices [39-41]. Here we use the variation of the soliton core size with polariton density to investigate the polariton nonlinearity in the $\mathrm{cw}$ regime. The waveguide geometry allows the accurate determination of the absolute polariton density inside the waveguide [42]. We thus deduce an interaction constant more than an order of magnitude larger than previously observed in the picosecond pulsed regime [2]. Using a numerical model of coherently coupled waveguide photons and excitons and an incoherent reservoir generated by scattering of the excitons we self-consistently fit all experimental features using a single value of the interaction strength. This provides a quantitative measure of the absolute size of the polariton interactions whereas previous investigations of excitonic reservoirs considered the spin anisotropy $[33,43,44]$.

The sample used in this work is the same as that in Ref. $[2,45]$. A schematic of the experiment is shown in Fig. 1(a). Figure 1(b) shows the polariton dispersion with the avoided crossing between uncoupled photon and exciton modes, characterized by Rabi splitting $\hbar \Omega=9 \mathrm{meV}$. Experiments were performed at $10 \mathrm{~K}$. A cw laser beam was modified using amplitude or phase masks and then projected onto an input grating coupler [see Fig. 1(a)] [2,45]. The input transverse profile was a $29 \mu \mathrm{m}$ FWHM Gaussian with either a phase jump or intensity dip near the center, corresponding to the two classes of initial conditions we investigate [46]. The polariton fluid undergoes nonlinear
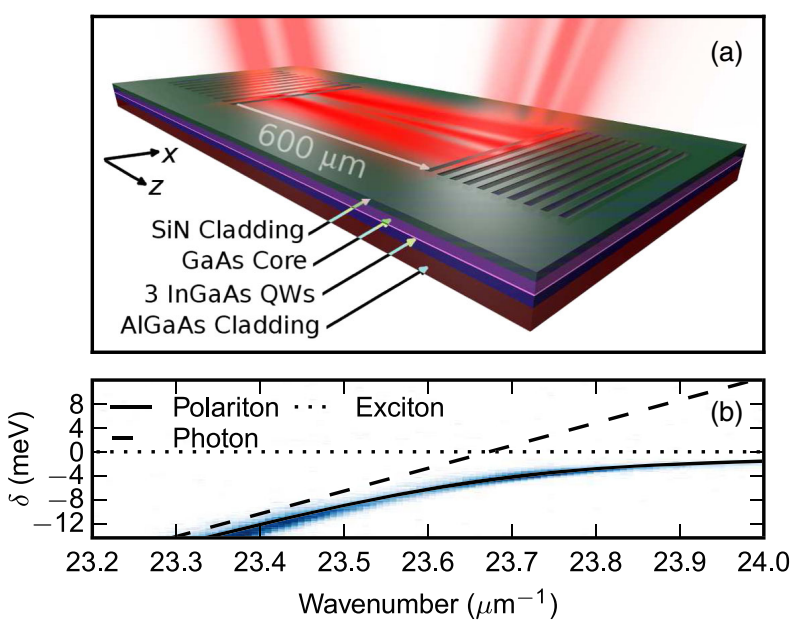

FIG. 1. (a) Schematic of polariton waveguide, and (b) lower polariton dispersion relation seen in the angle resolved photoluminescence spectrum.

evolution in a $600 \mu \mathrm{m}$ unpatterned region of planar waveguide and the light was collected by a second grating coupler and imaged onto a CCD camera.

We first consider the case of the phase jump initial condition. Figure 2(a) shows the intensity profile of the incident beam and the beam after propagation through the waveguide in the linear and nonlinear regimes. The input profile is Gaussian with a narrow dark notch near the center. At low excitation powers the nonlinearity is negligible and the diffraction of the discontinuous phase in the input field
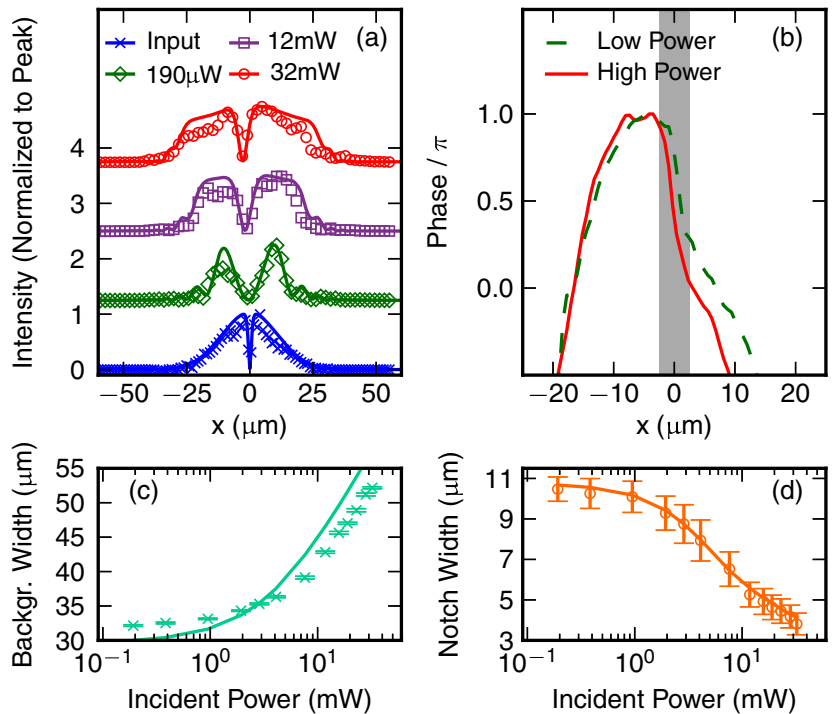

FIG. 2. Dark-soliton formation from the phase jump initial condition. (a) Experimental (points) and theoretical (full lines) intensity profiles of input field and output fields at three powers. (b) Experimental output phase at low and high power. [(c) and (d)] Experimental (points) and theoretical (full lines) power dependences of full width at one third maximum of background and FWHM of dark notches. 
results in a broad dip in the center of the Gaussian background [46]. The background itself is wide enough that it does not experience significant diffraction over the $600 \mu \mathrm{m}$ propagation length. Above $\sim 2 \mathrm{~mW}$ incident power the background broadens under the influence of nonlinear self-defocusing [4] while the notch width decreases, eventually forming a single narrow notch in a broad bright background, which is the expected profile for a single dark soliton [11]. Figure 2(b) shows a typical phase profile of the output field measured in a separate experiment for low and high power. For both powers a phase jump near $x=0$ is superimposed on a slowly varying background phase arising from the Gaussian background. The shaded region indicates the FWHM of the intensity notch at high power. The phase jump at high power is close to the value of $\pi$ injected at the input [46], as expected in the case of a single dark soliton [11]. Figures (c) and (d) show the pump power dependences of the widths of the background and of the dark notch. Crucially, the width of the notch narrows significantly as the density increases. This width-density dependence is exactly the trend expected for dark solitons and is the key evidence for their formation. Later we show that the rate of decrease with power is consistent with the size of the polariton nonlinearity in the system so that the width-density criterion for dark-soliton observation is quantitatively satisfied.

We now consider the amplitude defect initial condition. The field profiles are shown in Fig. 3(a). The intensity notch is clearly seen in the input field. At high density a pair of well-defined dark notches is present on the broad bright background of the output field. Figure 3(b) shows the difference in output phases between low and high power cases. An important evidence of dark-soliton formation is that there is a modification of the phase profile at high densities such that two phase jumps of opposite sign occur
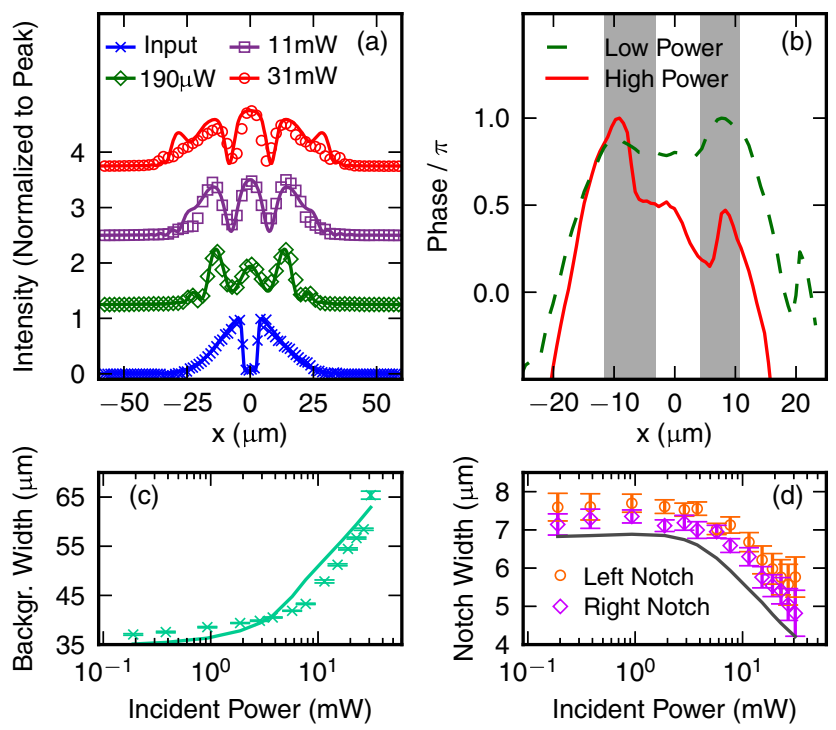

FIG. 3. As Fig. 2 for the amplitude defect initial condition. at the positions of the dark notches (shaded grey) that are not present at low power. This shows that the nonlinear interaction has generated the correct phase profile for a pair of dark solitons seeded by an intensity defect in the initial condition [11]. The relative depth of the dark notches in the intensity also increases at higher densities, consistent with the increased size of the phase jumps. As shown in Fig. 3(d), the notch widths decrease with increasing density, which, as for the phase defect, satisfies the width-density criterion for observation of dark solitons. Thus, we satisfy the criterion for multiple defect types and do so over a range of densities, providing conclusive evidence of soliton formation.

We now consider the origin and size of the nonlinear interaction responsible for generating the solitons. Figure 4(a) shows the measured output power from the waveguide vs the incident power, which is linear up to $10 \mathrm{~mW}$ where the narrowing of the dark notches and broadening of the background is already pronounced. From the gradient and known losses [2] we determine that $20 \pm$ $2 \%$ of the incident light couples into the guided mode [42].

The soliton width-density dependence formula Eq. (1) may be used to deduce an order of magnitude estimate of the effective size of the polariton-polariton interaction constant $g$ using the peak polariton density $n$ and soliton width $X_{0}$, both measured at the waveguide output. Values of $g$ for several powers are shown in Fig. 4(b) and are in the range $25-37 \mu \mathrm{eV} \mu \mathrm{m}^{2}$ for powers where the output vs input power is linear. The amplitude and phase defects give values that agree to within a factor of 2 . The difference arises because Eq. (1), strictly valid for homogeneous backgrounds, becomes modified for the amplitude defect where the solitons are on a region of finite slope [16]. Since there is no analytic expression for the corrections we use the unmodified Eq. (1) here but provide a full numerical treatment later in the paper. We see that the value of $g$ found from the numerical treatment, which does not assume soliton behavior, agrees with that found here using Eq. (1). This verifies that the dark notches satisfy the width-density dependence quantitatively as well as qualitatively, providing further evidence that they are dark solitons. We note that for a dark soliton with width $X_{0}=$ $7 \mu \mathrm{m}$ the balanced nonlinear and diffraction lengths are
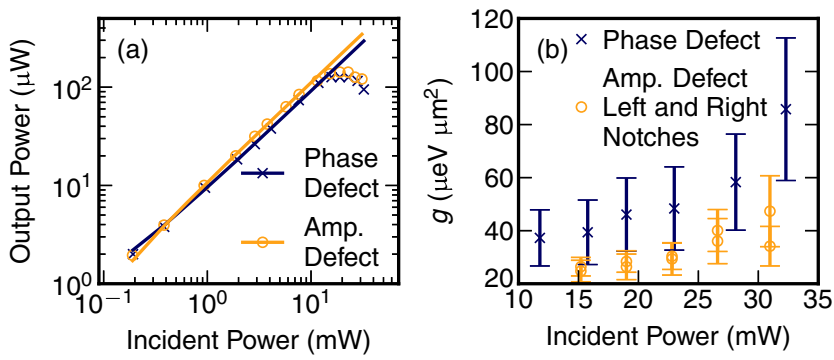

FIG. 4. (a) Output vs incident power for the two initial conditions. (b) Nonlinearity deduced from soliton width. 
equal to $\beta\left(X_{0} / 1.7626\right)^{2}=370 \mu \mathrm{m}$, which is less than the device length $L=600 \mu \mathrm{m}$. We can therefore be sure that solitons narrower than this are able to form in our device [27].

The values of $g$ we obtain are more than 2 orders of magnitude larger than $g=0.3 \mu \mathrm{eV} \mu \mathrm{m}^{2}$, which we previously deduced for dark and bright solitons using very similar structures but with picosecond pulses [2]. Scaling to account for the different detuning $\delta=-7.6 \mathrm{meV}$ in those measurements gives $g=1.5 \mu \mathrm{eV} \mu \mathrm{m}^{2}$, still over an order of magnitude less than we see here. This may occur if some of the polaritons are transferred into an excitonic reservoir with a lifetime long compared to the picosecond pulses. In the steady state the reservoir population will be larger than that of strongly coupled excitons by the ratio of its lifetime to excitation time, providing an excess exciton population that makes the polariton interaction appear stronger than it really is. The time scale of picosecond pulses is short compared to the reservoir excitation rate so no significant extra population builds up and only the strongly coupled exciton population contributes to the nonlinearity. The reservoir may be generated by scattering into excitonic states in the tail of the inhomeogeneously broadened exciton line $[47,48]$, for which the density of states is much larger than for polaritons. Alternatively, the reservoir can be composed of localized, indirect, or otherwise dark excitons [43,44,49-51]. We support the idea of a time-scale-dependent interaction constant with additional time-resolved experiments presented in Supplemental Material [52].

We now consider a numerical model that provides a self-consistent fit to all features of the experimental data. Polaritons propagating in the waveguide are described by Eqs. (2) for the slowly varying coupled photon and exciton envelope amplitudes $A$ and $\psi$ and the reservoir density $n_{R}$.

$$
\begin{gathered}
{\left[i \frac{\partial}{\partial t}+i \gamma_{p}+v_{\mathrm{g}}\left(i \frac{\partial}{\partial z}+\frac{1}{2 \beta_{e}} \frac{\partial^{2}}{\partial x^{2}}\right)\right] A=\left(\frac{\Omega}{2}\right) \psi,} \\
{\left[i \frac{\partial}{\partial t}+i\left(\gamma_{e}+\gamma_{r}\right)-g_{x}\left(|\psi|^{2}+n_{R}\right)\right] \psi=\left(\frac{\Omega}{2}\right) A,} \\
\frac{\partial n_{R}}{\partial t}=2 \gamma_{r}|\psi|^{2}-2 \gamma_{R} n_{R} .
\end{gathered}
$$

Here, $v_{\mathrm{g}}=58 \mu \mathrm{mps}^{-1}$ and $\beta_{e}=23.6 \mu \mathrm{m}^{-1}$ are the photon group velocity and wave number at the exciton frequency extracted from the fit to the dispersion relation in Fig. 1(b). The loss rates $\gamma_{p}$ and $\gamma_{r}$ are due to photon tunneling through the cladding and loss of excitons due to scattering to the reservoir while $\gamma_{e}$ quantifies all other exciton loss channels. The reservoir decay rate is $\gamma_{R}$. The total homogeneous exciton linewidth $\hbar\left(\gamma_{e}+\gamma_{r}\right)=$ $13.2 \mu \mathrm{eV}$ and $\hbar \gamma_{p}=32.9 \mu \mathrm{eV}$ were obtained from an independent fit to the spectral dependence of the loss length [53]. The (polarization averaged) exciton interaction energy per unit exciton density in one QW is given by $\hbar g_{x}$.

In the steady state, where $\partial n_{R} / \partial t=0$, Eq. (2c) can be rearranged to give $n_{R}=\left(\gamma_{r} / \gamma_{R}\right)|\psi|^{2}$. Substituting this into Eq. (2), the nonlinearity $g_{x}\left(|\psi|^{2}+n_{R}\right)$ becomes $g_{\text {eff }}|\psi|^{2}$ where $g_{\text {eff }}=g_{x}\left(1+\gamma_{r} / \gamma_{R}\right)$ is an effective exciton-exciton scattering that accounts for the fact that for every strongly coupled exciton the reservoir contains another $\gamma_{r} / \gamma_{R}$ incoherent excitons. For $\mathrm{cw}$ driving we use the ansatz $A=A(x, z) \exp (-i \delta t)$, and likewise for $\psi$, and eliminate $\psi$ using Eq. (2b) to leave a generalized GPE for $\partial A(x, z) / \partial z$ in terms of $A$ [54]. This was solved using a standard splitstep Fourier method [4].

The model output is plotted as solid lines in Figs. 2 and 3. Good semiquantitative agreement is achieved with all intensity profiles [panels (a)] and with the dark notch and background widths [panels (c) and (d)] at all powers. The only adjustable parameter $\hbar g_{\text {eff }}=220 \mu \mathrm{eV} \mu \mathrm{m}^{2}$ fits all the above data for both initial conditions at once so the model provides a self-consistent explanation of all features. The effective polariton-polariton interaction $g$ corresponding to the above exciton-exciton interaction is obtained using $\hbar g=\hbar g_{\text {eff }}|X|^{4} / N_{w}=25 \mu \mathrm{eV} \mu \mathrm{m}^{2}$ where $|X|^{2}=0.58$ is the exciton fraction and $N_{w}=3$ is the number of QWs [29]. This value is in good agreement with $\hbar g=25-37 \mu \mathrm{eV} \mu \mathrm{m}^{2}$ found earlier by applying Eq. (1) to the measured notch widths and densities. Thus, as noted earlier, the notch width power dependence is in quantitative agreement with the width-density dark-soliton criterion. At elevated powers the $g$ inferred from Eq. (1) increases due to additional nonlinear processes that likely also cause the nonlinear loss evident in Fig. 4(a). We finally note that literature values for $\hbar g_{\text {eff }}$ vary between 2 and $1740 \mu \mathrm{eV} \mu \mathrm{m}^{2}$ [29,30,34,35,55], which suggests a strong sample dependence of the $\mathrm{cw}$ nonlinearity. The influence of the reservoir on the interactions is a likely possible explanation for this sample dependence.

In conclusion, we have shown dark-soliton formation in a high velocity polariton fluid in the paraxial waveguide system. We observe dark notches with associated phase discontinuities that clearly vary between the linear and high density regimes. We have studied two different defect types and fully characterized the behavior in the linear regime so the effect of the nonlinearity is clear. The width of the features has the expected quantitative dependence on the background fluid density for a range of defects and densities, fulfilling the criterion for conclusive observation of polariton dark solitons. The experimental soliton and background field dynamics for both initial conditions are well reproduced using a numerical model of coupled photon, exciton, and reservoir evolution equations. We deduce a $\mathrm{cw}$ polariton-polariton interaction strength more than an order of magnitude larger than on a picosecond time scale, which may be attributed to the interaction of polaritons with the reservoir. 
Data supporting this study are openly available from the University of Sheffield repository [56]

We acknowledge support from Engineering and Physical Sciences Research Council Program Grants No. EP/ J007544/1 and No. EP/N031776/1, European Research Council Advanced Grant EXCIPOL (Project ID 320570), and Leverhulme Trust Grant No. PRG-2013-339. D. V. S. acknowledges support from the ITMO University Visiting Professorship via the Government of Russia (Grant No. 074-U01) and Russian Foundation for Basic Research (16-52-150006). P. M. W. acknowledges helpful discussions with D. M. Whittaker.

*p.m.walker@sheffield.ac.uk

[1] Exciton Polaritons in Microcavities, edited by V. Timofeev and D. Sanvitto (Springer, New York, 2014).

[2] P. M. Walker, L. Tinkler, D. V. Skryabin, A. Yulin, B. Royall, I. Farrer, D. A. Ritchie, M. S. Skolnick, and D. N. Krizhanovskii, Nat. Commun. 6, 8317 (2015).

[3] P. M. Walker, L. Tinkler, M. Durska, D. M. Whittaker, I. J. Luxmoore, B. Royall, D. N. Krizhanovskii, M. S. Skolnick, I. Farrer, and D. A. Ritchie, Appl. Phys. Lett. 102, 012109 (2013).

[4] G. P. Agrawal, Nonlinear Fibre Optics (Academic Press, New York, 2001).

[5] M. C. Rechtsman, J. M. Zeuner, Y. Plotnik, Y. Lumer, D. Podolsky, F. Dreisow, S. Nolte, M. Segev, and A. Szameit, Nature (London) 496, 196 (2013).

[6] M. C. Rechtsman, J. M. Zeuner, A. Tünnermann, S. Nolte, M. Segev, and A. Szameit, Nat. Photonics 7, 153 (2013).

[7] S. V. Suchkov, A. A. Sukhorukov, J. Huang, S. V. Dmitriev, C. Lee, and Y. S. Kivshar, Laser Photonics Rev. 10, 177 (2016).

[8] S. Longhi, Laser Photonics Rev. 3, 243 (2009).

[9] P. E. Larré and I. Carusotto, Phys. Rev. A 92, 043802 (2015).

[10] T. Karzig, C.-E. Bardyn, N. H. Lindner, and G. Refael, Phys. Rev. X 5, 031001 (2015).

[11] Y.S. Kivshar and G.P. Agrawal, Optical Solitons (Academic Press, New York, 2003).

[12] Y. S. Kivshar and B. Luther-Davies, Phys. Rep. 298, 81 (1998).

[13] G. R. Allan, S. R. Skinner, D. R. Andersen, and A. L. Smirl, Opt. Lett. 16, 156 (1991).

[14] G. A. Swartzlander, Jr., D. R. Andersen, J. J. Regan, H. Yin, and A. E. Kaplan, Phys. Rev. Lett. 66, 1583 (1991).

[15] V. Shandarov, D. Kip, M. Wesner, and J. Hukriede, J. Opt. A 2, 500 (2000).

[16] Y. S. Kivshar and X. Yang, Opt. Commun. 107, 93 (1994).

[17] N. P. Proukakis, N. G. Parker, D. J. Frantzeskakis, and C. S. Adams, J. Opt. B 6, S380 (2004).

[18] D. J. Frantzeskakis, J. Phys. A 43, 213001 (2010).

[19] A. Amo, S. Pigeon, D. Sanvitto, V. G. Sala, R. Hivet, I. Carusotto, F. Pisanello, G. Leménager, R. Houdré, E. Giacobino, C. Ciuti, and A. Bramati, Science 332, 1167 (2011).

[20] G. Grosso, G. Nardin, F. Morier-Genoud, Y. Léger, and B. Deveaud-Plédran, Phys. Rev. Lett. 107, 245301 (2011).
[21] V. Goblot, H. S. Nguyen, I. Carusotto, E. Galopin, A. Lemaître, I. Sagnes, A. Amo, and J. Bloch, Phys. Rev. Lett. 117, 217401 (2016).

[22] S. Blair and K. Wagner, Appl. Opt. 38, 6749 (1999).

[23] P. Cilibrizzi, H. Ohadi, T. Ostatnicky, A. Askitopoulos, W. Langbein, and P. Lagoudakis, Phys. Rev. Lett. 113, 103901 (2014).

[24] A. Amo, J. Bloch, A. Bramati, I. Carusotto, C. Ciuti, B. Deveaud, E. Giacobino, G. Grosso, A. Kamchatnov, G. Malpuech, N. Pavloff, S. Pigeon, D. Sanvitto, and D. D. Solnyshkov, Phys. Rev. Lett. 115, 089401 (2015).

[25] P. Cilibrizzi, H. Ohadi, T. Ostatnicky, A. Askitopoulos, W. Langbein, and P. Lagoudakis, Phys. Rev. Lett. 115, 089402 (2015).

[26] K. Guda, M. Sich, D. Sarkar, P. M. Walker, M. Durska, R. A. Bradley, D. M. Whittaker, M. S. Skolnick, E. A. Cerda-Méndez, P. V. Santos, K. Biermann, R. Hey, and D. N. Krizhanovskii, Phys. Rev. B 87, 081309(R) (2013).

[27] See Supplemental Material http://link.aps.org/supplemental/ 10.1103/PhysRevLett.119.097403 Sec. S4 for more detailed discussion of soliton properties and the relationship between density and soliton width.

[28] We calculated the sound velocity using the formula from Ref. [19].

[29] A. S. Brichkin, S. I. Novikov, A. V. Larionov, V. D. Kulakovskii, M. M. Glazov, C. Schneider, S. Höfling, M. Kamp, and A. Forchel, Phys. Rev. B 84, 195301 (2011).

[30] S. R. K. Rodriguez, A. Amo, I. Sagnes, L. Le Gratiet, E. Galopin, A. Lemaître, and J. Bloch, Nat. Commun. 7, 11887 (2016).

[31] M. M. Glazov, H. Ouerdane, L. Pilozzi, G. Malpuech, A. V. Kavokin, and A. D'Andrea, Phys. Rev. B 80, 155306 (2009).

[32] M. Vladimirova, S. Cronenberger, D. Scalbert, K. V. Kavokin, A. Miard, A. Lemaître, J. Bloch, D. Solnyshkov, G. Malpuech, and A. V. Kavokin, Phys. Rev. B 82, 075301 (2010).

[33] A. V. Sekretenko, S. S. Gavrilov, and V. D. Kulakovskii, Phys. Rev. B 88, 195302 (2013).

[34] L. Ferrier, E. Wertz, R. Johne, D. D. Solnyshkov, P. Senellart, I. Sagnes, A. Lemaître, G. Malpuech, and J. Bloch, Phys. Rev. Lett. 106, 126401 (2011).

[35] Y. Sun, Y. Yoon, M. Steger, G. Liu, L. N. Pfeiffer, K. West, D. W. Snoke, and K. A. Nelson, arXiv:1508.06698.

[36] I. Carusotto and C. Ciuti, Rev. Mod. Phys. 85, 299 (2013).

[37] A. Verger, C. Ciuti, and I. Carusotto, Phys. Rev. B 73, 193306 (2006).

[38] I. Carusotto, D. Gerace, H. E. Tureci, S. De Liberato, C. Ciuti, and A. Imamoğlu, Phys. Rev. Lett. 103, 033601 (2009).

[39] R. O. Umucalılar and I. Carusotto, Phys. Rev. Lett. 108, 206809 (2012).

[40] M. Hafezi, M. D. Lukin, and J. M. Taylor, New J. Phys. 15, 063001 (2013).

[41] X. Chen, Z.-C. Gu, Z.-X. Liu, and X.-G. Wen, Science 338, 1604 (2012).

[42] See Supplemental Material http://link.aps.org/supplemental/ 10.1103/PhysRevLett.119.097403 Sec. S2 for details of how we determined the coupling efficiency.

[43] D. N. Krizhanovskii, G. Dasbach, A. A. Dremin, V. D. Kulakovskii, N. A. Gippius, M. Bayer, and A. Forschel, Solid State Commun. 119, 435 (2001). 
[44] D. Sarkar, S. S. Gavrilov, M. Sich, J. H. Quilter, R. A. Bradley, N. A. Gippius, K. Guda, V. D. Kulakovskii, M. S. Skolnick, and D. N. Krizhanovskii, Phys. Rev. Lett. 105, 216402 (2010).

[45] See Supplemental Material http://link.aps.org/supplemental/ 10.1103/PhysRevLett.119.097403 Sec. S1 for further experimental details.

[46] See Supplemental Material http://link.aps.org/supplemental/ 10.1103/PhysRevLett.119.097403 Sec. S5 for mathematical expressions for initial conditions and comparison with experiment.

[47] A. Vinattieri, J. Shah, T. C. Damen, D. S. Kim, L. N. Pfeiffer, M.Z. Maialle, and L. J. Sham, Phys. Rev. B 50, 10868 (1994).

[48] D. M. Whittaker, Phys. Rev. Lett. 80, 4791 (1998).

[49] J.-M. Ménard, C. Poellmann, M. Porer, U. Leierseder, E. Galopin, A. Lemaître, A. Amo, J. Bloch, and R. Huber, Nat. Commun. 5, 4648 (2014).
[50] D. W. Snoke, W. W. Rühle, K. Köhler, and K. Ploog, Phys. Rev. B 55, 13789 (1997).

[51] J. O. Tollerud, S. T. Cundiff, and J. A. Davis, Phys. Rev. Lett. 117, 097401 (2016).

[52] See Supplemental Material http://link.aps.org/supplemental/ 10.1103/PhysRevLett.119.097403 Sec. S6 for supporting experiments investigating the nonlinear response at intermediate time scales.

[53] See Supplemental Material http://link.aps.org/supplemental/ 10.1103/PhysRevLett.119.097403 Fig. S2(d) for a comparison of experimental and modeled losses.

[54] See Supplemental Material http://link.aps.org/supplemental/ 10.1103/PhysRevLett.119.097403 Sec. S3 for details of the derivation of the generalized GPE.

[55] See Supplemental Material http://link.aps.org/supplemental/ 10.1103/PhysRevLett.119.097403 Sec. S7 for more detailed discussion of literature values.

[56] https://doi.org/10.15131/shef.data.5271802. 